\title{
Research Article \\ Periodic Oscillations in a Chemostat Model with Two Discrete Delays
}

\author{
Tiansi Zhang, Xuehui Ji, and Bo Li \\ College of Science, University of Shanghai for Science and Technology, Shanghai 200093, China \\ Correspondence should be addressed to Tiansi Zhang; zhangts1209@163.com
}

Received 22 October 2014; Accepted 30 January 2015

Academic Editor: Daniele Fournier-Prunaret

Copyright (C) 2015 Tiansi Zhang et al. This is an open access article distributed under the Creative Commons Attribution License, which permits unrestricted use, distribution, and reproduction in any medium, provided the original work is properly cited.

Periodic oscillations of solutions of a chemostat-type model in which a species feeds on a limiting nutrient are considered. The model incorporates two discrete delays representing the lag in nutrient recycling and nutrient conversion. Through the study of characteristic equation associated with the linearized system, a unique positive equilibrium is found and proved to be locally asymptotically stable under some conditions. Meanwhile, a Hopf bifurcation causing periodic solutions is also obtained. Numerical simulations illustrate the theoretical results.

\section{Introduction}

The chemostat is very important to imitate ecosystem and is used extensively in microbiology and population biology for its measurable parameters and maneuverable experiments; see $[1,2]$, for example. Many models have been constructed based on chemostat-type equations such as the growth of plankton of unicellular algae and plasmid-bearing and plasmid-free competition; see [3-5] and the references therein. Amongst them, regeneration of nutrient decomposed by bacterial played an indispensable role for the stability and development of the nature and thus was taken into account; see [6-8], but no one treated it with a time delay. In fact, a delay in nutrient recycling on ecosystem always exists and is influenced by the change of temperature [9]. In view of this, Bischi [10] embedded this time delay and discussed its stabilizing effect for a chemostat model with oscillations. After that, He, Ruan and Wang et al. studied the global behaviors of a chemostat model with delayed nutrient recycling and periodically pulsed input in [11] and [12], respectively. Freedman and $\mathrm{Xu}$ [13] turned to consider a competition model with delayed nutrient recycling which generalized the problem in [14] and gave some persistence and extinction conditions for the competing populations. For some other excellent work about the delayed nutrient recycling, one may refer to [1520].
On the other hand, environment is a vital factor that affects the growth of microorganisms. When it changes, a lag phase of the growth would appear. In 1969, when studying the growth of Isochrysis galbana, Caperon [21] used a time lag in his model with alterable environment and his research well met the corresponding experiment results. Besides it, Ellermeyer [22] and Xia et al. [23] also investigated the delayed response in growth and got global asymptotic behaviors of a competition and transient oscillations, respectively.

From the above we see that time delays have biological significance since they can alter dynamics of solutions of a system and display more transient oscillations especially in numerical simulation. So in this paper, we confine us to a single species model with a limited nutrient at a constant rate together with two delays in nutrient recycling and nutrient conversion, which takes the following form:

$$
\begin{gathered}
\frac{d S}{d t}=D\left(S^{0}-S\right)-a P(S) x+\gamma D_{1} x\left(t-\tau_{2}\right), \\
\frac{d x}{d t}=a e^{-D \tau_{1}} P\left(S\left(t-\tau_{1}\right)\right) x\left(t-\tau_{1}\right)-D x-D_{1} x,
\end{gathered}
$$

with initial value conditions

$$
S(\theta)=\varphi(\theta) \geq 0, \quad x(\theta)=\psi(\theta) \geq 0,
$$

where $S(t)$ denotes the limiting nutrient concentration and $x(t)$ denotes the concentration of the microorganism at time 
t. $\varphi, \psi \in B C\left[-\max \left\{\tau_{1}, \tau_{2}\right\}, 0\right]$, the Banach space of all continuous bounded functions, and all parameters are positive constants. $S^{0}$ is the input concentration of the limiting nutrient, $D$ is the washout rate of the chemostat, $D_{1}$ is the death rate of the microorganisms, $\gamma \in(0,1)$ is the fraction of nutrient conversion, and $a$ is the maximal nutrient uptake rate for the microorganisms. There are two delays of $\tau_{1}$ and $\tau_{2}$ due to nutrient conversion and nutrient recycling, respectively. $P(S)$ is the nutrient uptake function and has the following properties:

$$
P(0)=0, \quad \frac{d S}{d t}>0, \quad \lim _{S \rightarrow \infty} P(S)=1 .
$$

This is the first time to study such a problem. The method used here is established by Ruan and Wei [24]. Our analysis will develop from discussing roots of the associated characteristic equation of the linearized system, whose coefficients depend heavily on delays.

The paper is organized as follows. In the next section, we investigate the existence of a positive equilibrium. Then in Section 3, we study the asymptotic stability of the equilibrium by first linearizing the system and then analyzing the roots of the associated characteristic equation, which is a second degree exponential polynomial. This process is divided into three cases of which two delays are zero, only one delay is zero, and no delays are zero. Finally a Hopf bifurcation causing the appearance of periodic solutions is found to exist. The last two sections are to numerically illustrate our findings and to summarize the discussion in the paper, respectively.

\section{The Boundedness and Existence of Nonnegative Equilibria of Model (1)}

Firstly, by studying model (1) with (3), we have the following result about the boundedness of solutions.

Theorem 1. All solutions of system (1) with (3) are bounded.

Proof. Define a Lyapunov function

$$
\begin{aligned}
V(t, S, x)= & S(t)+e^{D \tau_{1}} x \\
& +\int_{t-\tau_{1}}^{t} a P(S(u)) x(u) d u \\
& +\gamma D_{1} \int_{t-\tau_{2}}^{t} x(u) d u .
\end{aligned}
$$

Obviously, $V \geq 0$ and $V \rightarrow+\infty$ as $|(S, x)| \rightarrow+\infty$. Differentiate $V$ along trajectories of (1); that is,

$$
\begin{aligned}
\dot{V}= & D\left(S^{0}-S\right)-a P(S) x+\gamma D_{1} x\left(t-\tau_{2}\right) \\
& +e^{D \tau_{1}}\left(a e^{-D \tau_{1}} P\left(S\left(t-\tau_{1}\right)\right) x\left(t-\tau_{1}\right)-D x-D_{1} x\right)
\end{aligned}
$$

$$
\begin{aligned}
& +a\left(P(S) x-P\left(S\left(t-\tau_{1}\right)\right) x\left(t-\tau_{1}\right)\right) \\
& +\gamma D_{1}\left(x(t)-x\left(t-\tau_{2}\right)\right) \\
= & D\left(S^{0}-S\right)-\left(\left(D+D_{1}\right) e^{D \tau_{1}}-\gamma D_{1}\right) x .
\end{aligned}
$$

It is easy to see that the coefficient of $x$ is negative since $\gamma \in$ $(0,1)$. So outside the region bounded by the positive orthant and the line

$$
x=\frac{D\left(S^{0}-S\right)}{\left(D+D_{1}\right) e^{D \tau_{1}}-\gamma D_{1}},
$$

$\dot{V}$ is negative. This completes the proof.

Secondly, note that $E_{0}=\left(S^{0}, 0\right)$ is always an equilibrium for system (1), and from the following equations

$$
\begin{gathered}
D\left(S^{0}-S^{*}\right)=\left(a P\left(S^{*}\right)-\gamma D_{1}\right) x^{*}, \\
a P\left(S^{*}\right) x^{*}=\left(D+D_{1}\right) e^{D \tau_{1}} x^{*}
\end{gathered}
$$

system (1) has a positive equilibrium $E^{*}\left(S^{*}, x^{*}\right)$ given by

$$
\begin{gathered}
S^{*}=P^{-1}\left(\frac{D+D_{1}}{a} e^{D \tau_{1}}\right), \\
x^{*}=\frac{D\left(S^{0}-S^{*}\right)}{a P\left(S^{*}\right)-\gamma D_{1}},
\end{gathered}
$$

provided that

$$
\frac{D+D_{1}}{a} e^{D \tau_{1}}<P\left(S^{0}\right)
$$

where $P^{-1}$ means the inverse function of $P$. Moreover, since the function $P(S)$ is increasing, the equilibrium $E^{*}$ is unique. So we can conclude the following.

Theorem 2. System (1) has a unique trivial equilibrium $E_{0}$, and has a unique positive equilibrium $E^{*}$ defined by (8) if condition (9) holds.

\section{Asymptotic Stability of $E^{*}$}

In this section, we are going to discuss the asymptotic stability of the positive equilibrium $E^{*}$ of system (1). Throughout this section, we assume that condition (9) holds. To begin we linearize (1) at $E^{*}$ and then construct the associated characteristic equation.

Setting $z_{1}=S-S^{*}$ and $z_{2}=x-x^{*}$ and putting them into system (1), we have

$$
\begin{aligned}
\frac{d z_{1}}{d t}= & -\left(D+a x^{*} P^{\prime}\left(S^{*}\right)\right) z_{1}-a P\left(S^{*}\right) z_{2} \\
& +\gamma D_{1} z_{2}\left(t-\tau_{2}\right), \\
\frac{d z_{2}}{d t}= & -\left(D+D_{1}\right) z_{2}+a x^{*} P^{\prime}\left(S^{*}\right) e^{-D \tau_{1}} z_{1}\left(t-\tau_{1}\right) \\
& +a P\left(S^{*}\right) e^{-D \tau_{1}} z_{2}\left(t-\tau_{1}\right) .
\end{aligned}
$$


Denote

$$
\begin{aligned}
& A_{0}=\left(\begin{array}{cc}
-\left(D+a x^{*} P^{\prime}\left(S^{*}\right)\right) & -a P\left(S^{*}\right) \\
0 & -\left(D+D_{1}\right)
\end{array}\right), \\
& A_{1}=\left(\begin{array}{cc}
0 & 0 \\
a x^{*} P^{\prime}\left(S^{*}\right) e^{-D \tau_{1}} & a P\left(S^{*}\right) e^{-D \tau_{1}}
\end{array}\right), \\
& A_{2}=\left(\begin{array}{cc}
0 & \gamma D_{1} \\
0 & 0
\end{array}\right) .
\end{aligned}
$$

Then the characteristic equation associated with (10) is given by

$$
\operatorname{det}\left(\lambda I-A_{0}-e^{-\lambda \tau_{1}} A_{1}-e^{-\lambda \tau_{2}} A_{2}\right)=0,
$$

or equivalently by the second degree exponential polynomial in $\lambda$,

$$
\begin{aligned}
\lambda^{2} & +\left(\left(D+a x^{*} P^{\prime}\left(S^{*}\right)\right)+\left(D+D_{1}\right)\right) \lambda \\
& +\left(D+D_{1}\right)\left(D+a x^{*} P^{\prime}\left(S^{*}\right)\right) \\
& -a P\left(S^{*}\right) e^{-D \tau_{1}}(\lambda+D) e^{-\lambda \tau_{1}} \\
& -a \gamma D_{1} e^{-D \tau_{1}} x^{*} P^{\prime}\left(S^{*}\right) e^{-\lambda\left(\tau_{1}+\tau_{2}\right)}=0 .
\end{aligned}
$$

From now we restrict our attention to discuss the local asymptotic stability of $E^{*}$ by analyzing the sign of the real parts of roots of the characteristic equation (13). Note that the coefficients of (13) depend heavily on the time delays $\tau_{1}$ and $\tau_{2}$; there is not a direct way to go, so by use of the method proposed by Ruan and Wei in [24], we consider three cases: $\tau_{1}=0, \tau_{2}=0 ; \tau_{1}=0, \tau_{2}>0$; and $\tau_{1}>0, \tau_{2}>0$.

3.1. The Case $\tau_{1}=\tau_{2}=0$. Assume in (13) that $\tau_{1}=\tau_{2}=0$; then we have

$$
\begin{aligned}
& \lambda^{2}+\left(D+a x^{*} P^{\prime}\left(S^{*}\right)\right) \lambda \\
& \quad+a x^{*} P^{\prime}\left(S^{*}\right)\left(a P\left(S^{*}\right)-\gamma D_{1}\right)=0 .
\end{aligned}
$$

According to the Routh-Hurwith criterion, the real parts of all eigenvalues of (14) are negative if and only if (9) is valid, which implies the asymptotic stability of $E^{*}$. Hence, we have the following result.

Theorem 3. Assume that condition (9) and $\tau_{1}=\tau_{2}=0$ hold. Then all roots of (13) have negative real parts, and the positive equilibrium $E^{*}$ of system (1) is locally asymptotically stable.

3.2. The Case $\tau_{1}=0, \tau_{2}>0$. Setting $\tau_{1}=0$ in (13), since $a P\left(S^{*}\right)=D+D_{1}$, the characteristic equation is now

$$
\lambda^{2}+A \lambda+B-C e^{-\lambda \tau_{2}}=0,
$$

where

$$
\begin{gathered}
A=D+a x^{*} P^{\prime}\left(S^{*}\right), \\
B=a^{2} x^{*} P\left(S^{*}\right) P^{\prime}\left(S^{*}\right), \\
C=\gamma D_{1} a x^{*} P^{\prime}\left(S^{*}\right) .
\end{gathered}
$$

It is not hard to see that $A>0$ and $B>C>0$.
We know from Theorem 3 that the $E^{*}$ is locally asymptotically stable if set $\tau_{2}=0$ in (15), while when $\tau_{2}$ increases from zero, the equilibrium could lose its stability if pure imaginary roots of (13) appear. Therefore it is convenient to look for pure imaginary roots of (15).

Put $\lambda=i \omega, \omega \neq 0 \in \mathbb{R}$ into (15) and separate the real and the imaginary parts of the equation; then we have

$$
\cos \left(\omega \tau_{2}\right)=\frac{B-\omega^{2}}{C}, \quad \sin \left(\omega \tau_{2}\right)=-\frac{A \omega}{C} .
$$

Taking the sum of squares in (17) yields

$$
\omega^{4}+\left(A^{2}-2 B\right) \omega^{2}+B^{2}-C^{2}=0 .
$$

It has real solutions

$$
\omega_{ \pm}^{2}=\frac{1}{2}\left(2 B-A^{2} \pm \sqrt{\left(2 B-A^{2}\right)^{2}-4\left(B^{2}-C^{2}\right)}\right),
$$

provided

$$
\left(A^{2}-2 B\right)^{2}>4\left(B^{2}-C^{2}\right)>0>A^{2}-2 B .
$$

Notice that if $\omega$ is a solution of (17), so is $-\omega$. Accordingly (15) has two pure imaginary solutions $\lambda_{ \pm}=i \omega_{ \pm}$with $\omega_{+}>\omega_{-}>0$.

Now differentiate both sides of (15) with respect to $\tau_{2}$,

$$
\left(2 \lambda+A+C \tau_{2} e^{-\lambda \tau_{2}}\right) \frac{d \lambda}{d \tau_{2}}=-C \lambda e^{-\lambda \tau_{2}}
$$

and then

$$
\begin{aligned}
\left(\frac{d \lambda}{d \tau_{2}}\right)^{-1} & =-\frac{(2 \lambda+A) e^{\lambda \tau_{2}}}{C \lambda}-\frac{\tau_{2}}{\lambda}, \\
e^{\lambda \tau_{2}} & =\frac{C}{\lambda^{2}+A \lambda+B} .
\end{aligned}
$$

Therefore,

$$
\begin{aligned}
\operatorname{sign} & \left\{\frac{d(\operatorname{Re} \lambda)}{d \tau_{2}}\right\}_{\lambda=i \omega} \\
& =\operatorname{sign}\left\{\operatorname{Re}\left(\frac{d \lambda}{d \tau_{2}}\right)^{-1}\right\}_{\lambda=i \omega} \\
& =\operatorname{sign}\left\{\operatorname{Re}\left(-\frac{2 \lambda+A}{\lambda\left(\lambda^{2}+A \lambda+B\right)}\right)_{\lambda=i \omega}\right\} \\
& =\operatorname{sign}\left\{\frac{A^{2}-2\left(B-\omega^{2}\right)}{A^{2} \omega^{2}+\left(B-\omega^{2}\right)^{2}}\right\} \\
& =\operatorname{sign}\left\{A^{2}-2 B+2 \omega^{2}\right\} .
\end{aligned}
$$

Clearly the sign of $d(\operatorname{Re} \lambda) / d \tau_{2}$ is positive at $\omega_{+}$or is negative at $\omega_{-}$.

Furthermore, we see that there are two sets of $\tau_{2}$ satisfying (17):

$$
\tau_{2,1}^{n}=\frac{\theta_{1}}{\omega_{+}}+\frac{2 n \pi}{\omega_{+}}
$$


where $0 \leq \theta_{1}<2 \pi, n=1,2,3, \ldots$, and

$$
\begin{gathered}
\cos \theta_{1}=\frac{B-\omega_{+}^{2}}{C}, \quad \sin \theta_{1}=-\frac{A \omega_{+}}{C}, \\
\tau_{2,2}^{n}=\frac{\theta_{2}}{\omega_{-}}+\frac{2 n \pi}{\omega_{-}},
\end{gathered}
$$

where $0 \leq \theta_{2}<2 \pi$, and

$$
\cos \theta_{2}=\frac{B-\omega_{-}^{2}}{C}, \quad \sin \theta_{2}=-\frac{A \omega_{-}}{C} .
$$

To sum up, we have the following.

Lemma 4. Let $\tau_{2, i}^{*}$ be an element of the sequences $\left\{\tau_{2, i}^{n}\right\}, i=1,2$. For $\tau_{2}=\tau_{2, i}^{*}$, the characteristic equation (15) has a pair of simple conjugate pure imaginary roots $\pm i \omega_{ \pm}$, satisfying

$$
\operatorname{sign}\left\{\frac{d(\operatorname{Re} \lambda)}{d \tau_{2}}\right\}_{\tau=\tau_{2, i}^{*}}=\operatorname{sign}\left\{A^{2}-2 B+2 \omega_{ \pm}^{2}\right\} .
$$

From the above analysis, the stability of the positive equilibrium $E^{*}$ of system (1) for $\tau_{1}=0$ is obvious.

Theorem 5. Assume that conditions (9) and (20) hold and $\tau_{1}=$ 0 . Let

$$
\tau_{2}^{*}=\min _{i=1,2 ; n \in N}\left\{\tau_{2, i}^{n}\right\}
$$

Then the equilibrium $E^{*}$ of system (1) is locally asymptotically stable when $\tau<\tau_{2}^{*}$ and a Hopf bifurcation occurs at $E^{*}$ when $\tau=\tau_{2}^{*}$ if and only if $\operatorname{sign}\left\{A^{2}-2 B+2 \omega_{ \pm}^{2}\right\}>0$.

3.3. The Case $\tau_{1}, \tau_{2}>0$. The case $\tau_{1}, \tau_{2}>0$ can be regarded as a general one of that in the last section, so our discussion here is based on the result in Section 3.2. The asymptotic stability of $E^{*}$ can be derived from Theorem 5 . To state it, we first introduce a lemma about the sign of the real parts of roots of the characteristic equation (13).

Lemma 6. If all roots of (15) have negative real parts for $\tau_{2}>0$, then there exists a $\tau_{1}^{*}\left(\tau_{2}\right)>0$, such that all roots of (13) have negative real parts for $\tau_{1} \in\left[0, \tau_{1}^{*}\left(\tau_{2}\right)\right)$.

Proof. Suppose that (15) has no roots with nonnegative real parts for $\tau_{2}>0$; that is, all the roots of (13) have negative real parts for $\tau_{1}=0$ and $\tau_{2}>0$. According to Theorem 2.1 in [21], on the left side of (13), the sum of the multiplicity of zeros may change if a zero locates on or passes through the imaginary axis in the open right half-plane when $\tau_{1}$ varies.

Recall that (13) with $\tau_{1}=0$ has no root with nonnegative real part; then there must exist a $\tau_{1}^{*}\left(\tau_{2}\right)>0$ such that all roots of (13) have negative real parts for $\tau_{1}<\tau_{1}^{*}\left(\tau_{2}\right)$.

Theorem 7. Assume that conditions (9) and (20) hold. Then for any $\tau_{2} \in\left[0, \tau_{2}^{*}\right)$, where $\tau_{2}^{*}$ is defined in Theorem 5 , there exists a $\tau_{1}^{*}\left(\tau_{2}\right)>0$ such that the positive equilibrium $E^{*}$ of system (1) is locally asymptotically stable when $\tau_{1} \in\left[0, \tau_{1}^{*}\left(\tau_{2}\right)\right.$ ).
Proof. From Theorem 5, it follows that all roots of (15) have negative real parts when $\tau_{2} \in\left[0, \tau_{2}^{*}\right)$. Then Lemma 6 indicates the validity of the conclusion.

\section{Numerical Simulations}

In this section, we give some numerical tests to support our findings in the previous section. Besides this, we observe the existence of periodic solutions arising from the Hopf bifurcation.

In system (1), we assume that the nutrient uptake function $P(S)$ is of Michaelis-Menten type

$$
P(S)=\frac{S}{k+S},
$$

where $k>0$ is the half-saturation constant $[25,26]$. We take the parameters $D=0.01, D_{1}=0.5, \gamma=0.8, k=0.1, a=1$, and $S^{0}=1$. Figure 1 shows the existence and the stability of $E^{*}$ for the case $\tau_{1}=\tau_{2}=0$.

For $\tau_{1}=0$ and $\tau_{2}<\tau_{2}^{*}$, Figure 2 implies the asymptotical stability of $E^{*}$ as stated in Theorem 5 . Moreover there also exists the phenomenon of damped oscillations about the equilibrium.

Notice that, in Theorem 5, a Hopf bifurcation exists for $\tau_{1}=0$ and $\tau_{2}=\tau_{2}^{*}$. It is natural to find that, for $\tau_{1}=0$ and $\tau_{2}>\tau_{2}^{*}$, the oscillating solutions emerge; please see Figure 3 . Besides, we can see that the periodic oscillation is stable.

Meanwhile, we can still expect to obtain the existence of the steady state $E^{*}$ by taking $\tau_{1} \leq 57.8$ under condition (9).

Figure 4 presents the local asymptotic stability of $E^{*}$ for $\tau_{1}=0.9$ and $\tau_{2}=12.217$ which is in accordance with the result in Theorem 7 .

\section{Discussion}

The most simple idealization for an ecosystem is probably the chemostat, of which the imitation is very close to that in the natural environment such as lakes. But the real environment is more complicated than a chemostat and the continuous culture of microorganisms can be affected by multiple factors, for instance, temperature, climate, food, and so forth. In addition, the nutrient recycling and absorption are also crucial to microorganisms in nature particularly with variable environment. Based on these, many models of chemostat systems have been developed; see $[16,17]$ for the models with delayed nutrient recycling and $[19,27,28]$ with delayed response in growth, respectively.

In this paper, we have investigated a chemostat model of a single species with inhibitory exponential substrate uptake and two discrete delays. One indicates the lag caused by gestation; the other models the partially recycled nutrient decomposed by bacteria after the death of biomass.

The characteristic equation of the linearized system at the nonzero equilibrium is defined by an exponential polynomial of second degree with delay-dependent coefficients. Because of the existence of two delays, general analytical methods to study the roots of this equation could not be used directly. In the paper, we carry out a rigorous analysis of the stability 

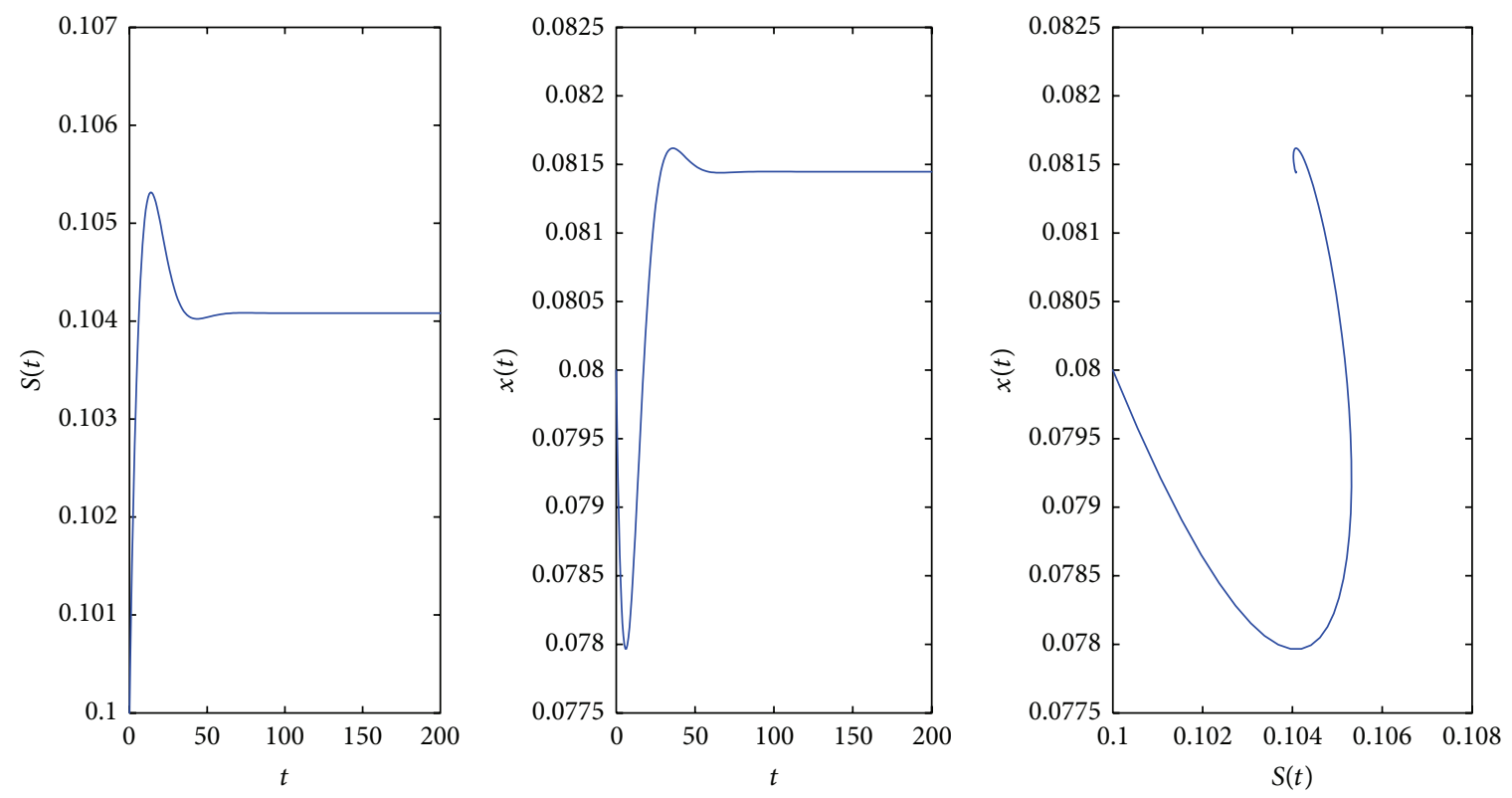

FIgURE 1: Asymptotic stability. The steady state $E^{*}$ is locally asymptotically stable. The parameters are $D=0.01, D_{1}=0.5, \gamma=0.8, k=0.1$, $a=1$, and $S^{0}=1$. The time delays are $\tau_{1}=0$ and $\tau_{2}=0$.
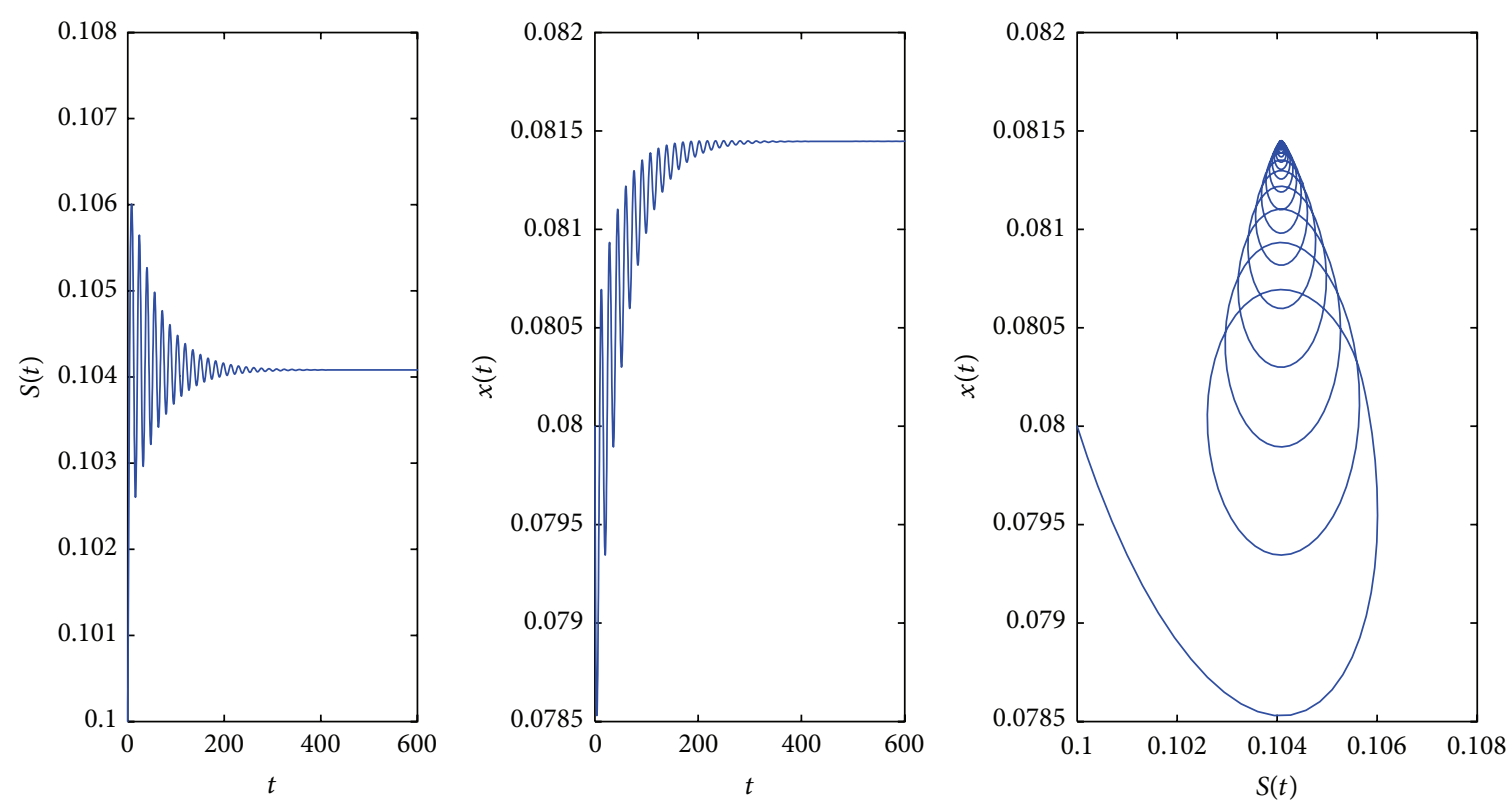

Figure 2: Asymptotic stability. The steady state $E^{*}$ of (1) is locally asymptotically stable, even though damped oscillations can be observed. The parameters are $D=0.01, D_{1}=0.5, \gamma=0.8, k=0.1, a=1$, and $S^{0}=1$. The time delays are $\tau_{1}=0$ and $\tau_{2}=10$.

of the model. Firstly we concentrate on the case when one of the delays, $\tau_{1}$, equals zero and obtains a critical value for the delay $\tau_{2}$ : when $\tau_{2}<\tau_{2}^{*}$ all roots of the characteristic equation have negative parts and when $\tau_{2}=\tau_{2}^{*}$ purely imaginary roots appear. Then fix the range $\tau_{2}<\tau_{2}^{*}$; we show that there exists a number $\tau_{1}^{*}\left(\tau_{2}\right)$ such that all roots of the characteristic equation have negative real parts for $\tau_{1} \in\left[0, \tau_{1}^{*}\left(\tau_{2}\right)\right]$. Consequently, the stability results for the chemostat model with two independent delays are obtained and the existence of a Hopf bifurcation that produces periodic solutions is also proven. To test our findings, some numerical simulations are demonstrated which present the periodic oscillations.

\section{Conflict of Interests}

The authors declare that there is no conflict of interests regarding the publication of this paper. 

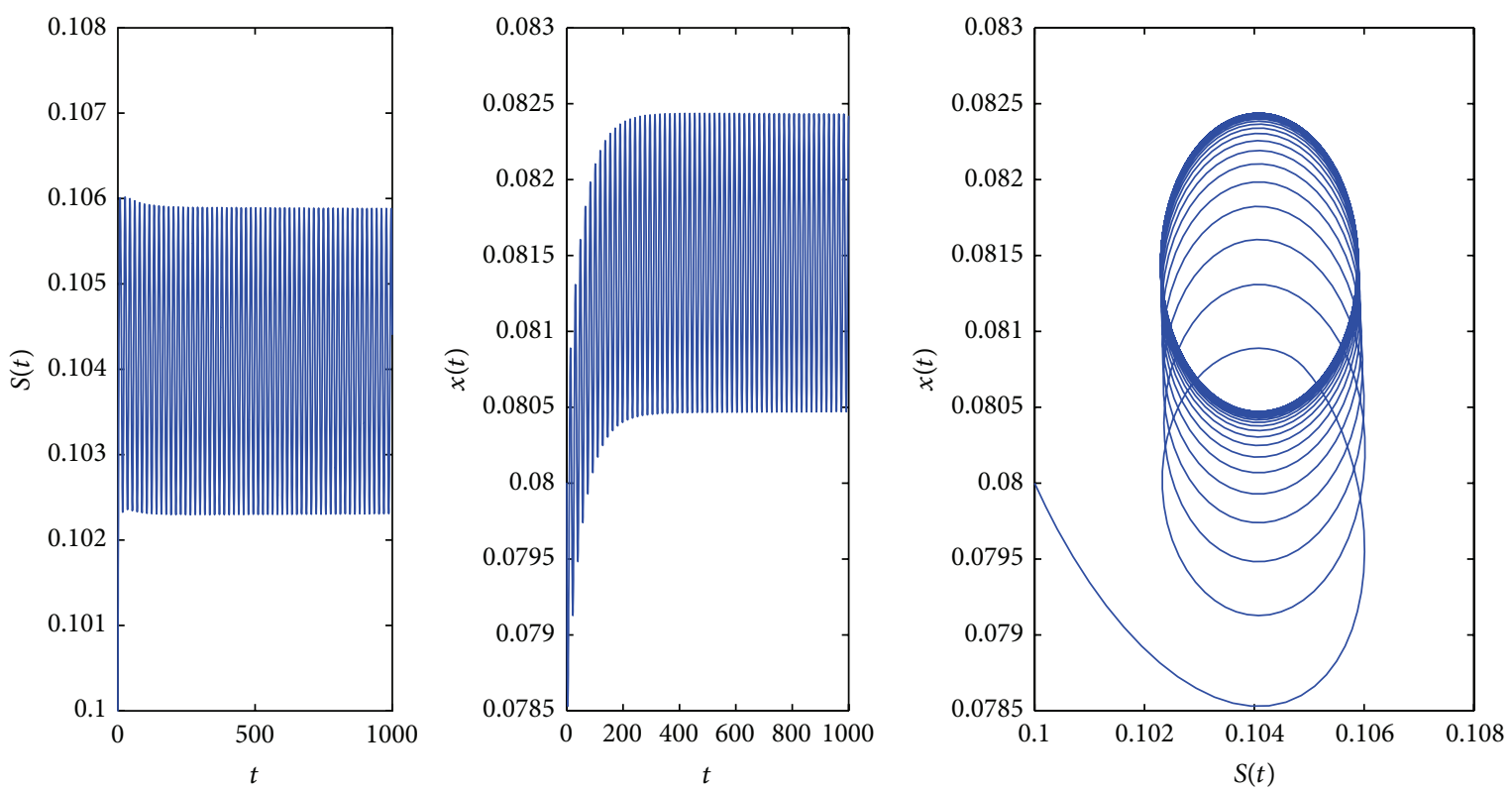

FIGURE 3: Oscillation solutions with $\tau_{1}=0$. Parameters are $D=0.01, D_{1}=0.5, \gamma=0.8, k=0.1, a=1$, and $S^{0}=1$. The time delay is $\tau_{2}=12.217$.
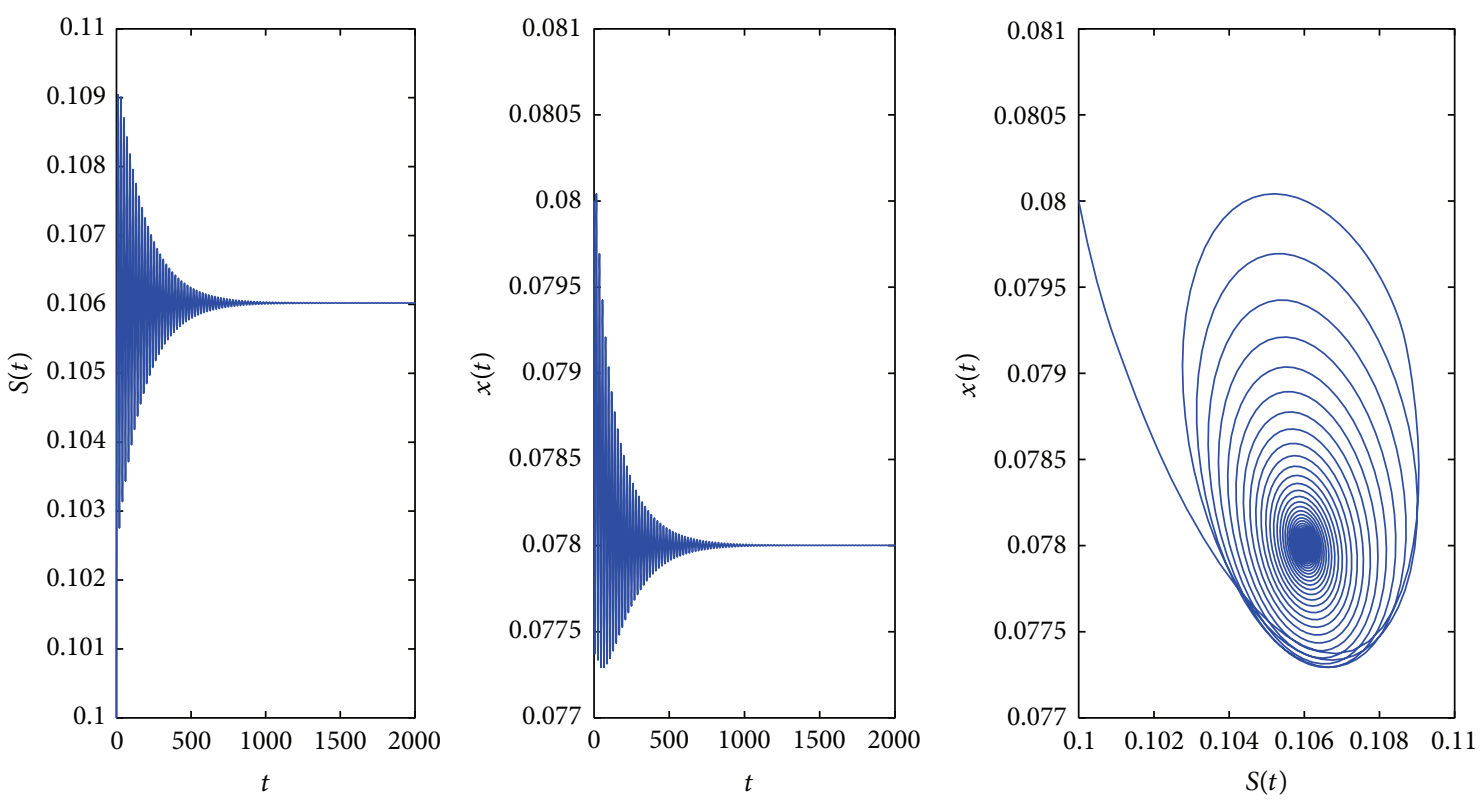

Figure 4: Asymptotic stability. The steady state $E^{*}$ of (1) is locally asymptotically stable, even though damped oscillations can be observed. Parameters are $D=0.01, D_{1}=0.5, \gamma=0.8, k=0.1, a=1$, and $S^{0}=1$. The time delays are $\tau_{1}=0.9$ and $\tau_{2}=12.217$.

\section{Acknowledgments}

The authors would like to acknowledge the support from the National Natural Science Foundation of China (11271260) and the Innovation Program of Shanghai Municipal Education Commission (13ZZ116).

\section{References}

[1] T. Egli, "The ecology and physiological significance of the growth of heterotrophicmicroorganisms with mixtures of substrates," in Advances in Microbial Ecology, vol. 14 of Advances in Microbial Ecology, pp. 305-386, Springer US, New York, NY, USA, 1995. 
[2] H. L. Smith and P. Waltman, The Theory of the Chemostat, vol. 13 of Cambridge Studies in Mathematical Biology, Cambridge University, Cambridge, UK, 1995.

[3] S. B. Hsu, S. Hubbell, and P. Waltman, "A mathematical theory for single-nutrient competition in continuous cultures of micro-organisms," SIAM Journal on Applied Mathematics, vol. 32, no. 2, pp. 366-383, 1977.

[4] P. Waltman, S. P. Hubbell, and S. B. Hsu, "Theoretical and experimental investigations of microbial competition in continuous culture," in Modeling and Differential Equations in Biology, T. Burton, Ed., pp. 107-152, Dekker, New York, NY, USA, 1980.

[5] S. L. Yuan, D. M. Xiao, and M. A. Han, "Competition between plasmid-bearing and plasmid-free organisms in a chemostat with nutrient recycling and an inhibitor," Mathematical Biosciences, vol. 202, no. 1, pp. 1-28, 2006.

[6] R. M. Nisbet, J. McKinstry, and W. S. C. Gurney, "A 'strategic' model of material cycling in a closed ecosystem," Mathematical Biosciences, vol. 64, no. 1, pp. 99-113, 1983.

[7] S. G. Ruan, "Persistence and coexistence in zooplanktonphytoplankton-nutrient models with instantaneous nutrient recycling," Journal of Mathematical Biology, vol. 31, no. 6, pp. 633-654, 1993.

[8] S. G. Ruan, "A three-trophic-level model of plankton dynamics with nutrient recycling," The Canadian Applied Mathematics Quarterly, vol. 1, no. 4, pp. 529-553, 1993.

[9] R. H. Whittaker, Communities and Ecosystems, Macmillan, New York, NY, USA, 1975.

[10] G. I. Bischi, "Effects of time lags on transient characteristics of a nutrient cycling model," Mathematical Biosciences, vol. 109, no. 2, pp. 151-175, 1992.

[11] X.-Z. He and S. Ruan, "Global stability in chemostat-type plankton models with delayed nutrient recycling," Journal of Mathematical Biology, vol. 37, no. 3, pp. 253-271, 1998.

[12] K. Wang, Z. D. Teng, and F. Q. Zhang, "Global behaviors of a chemostat model with delayed nutrient recycling and periodically pulsed input," Discrete Dynamics in Nature and Society, vol. 2010, Article ID 830951, 21 pages, 2010.

[13] H. I. Freedman and Y. T. Xu, "Models of competition in the chemostat with instantaneous and delayed nutrient recycling," Journal of Mathematical Biology, vol. 31, no. 5, pp. 513-527, 1993.

[14] E. Beretta, G. I. Bischi, and F. Solimano, "Stability in chemostat equations with delayed nutrient recycling," Journal of Mathematical Biology, vol. 28, no. 1, pp. 99-111, 1990.

[15] S. G. Ruan and G. Wolkowicz, "Persistence in plankton models with delayed nutrient recycling," The Canadian Applied Mathematics Quarterly, vol. 3, no. 2, pp. 219-235, 1995.

[16] S. G. Ruan, "Oscillations in plankton models with nutrient recycling," Journal of Theoretical Biology, vol. 208, no. 1, pp. 1526, 2001.

[17] P. de Leenheer, B. Li, and H. L. Smith, "Competition in the chemostat: some remarks," Canadian Applied Mathematics Quarterly, vol. 11, no. 3, pp. 229-248, 2003.

[18] Z. Q. Lu and K. P. Hadeler, "Model of plasmid-bearing, plasmidfree competition in the chemostat with nutrient recycling and an inhibitor," Mathematical Biosciences, vol. 148, no. 2, pp. 147$159,1998$.

[19] S. Yuan and T. Zhang, "Dynamics of a plasmid chemostat model with periodic nutrient input and delayed nutrient recycling," Nonlinear Analysis: Real World Applications, vol. 13, no. 5, pp. 2104-2119, 2012.
[20] X.-Z. He, S. Ruan, and H. Xia, "Global stability in chemostattype equations with distributed delays," SIAM Journal on Mathematical Analysis, vol. 29, no. 3, pp. 681-696, 1998.

[21] J. Caperon, "Time lag in population growth response of isochrysis galbana to a variable nitrate environment," Ecology, vol. 50, no. 2, pp. 188-192, 1969.

[22] S. F. Ellermeyer, "Competition in the chemostat: global asymptotic behavior of a model with delayed response in growth," SIAM Journal on Applied Mathematics, vol. 54, no. 2, pp. 456465, 1994.

[23] H. Xia, G. S. Wolkowicz, and L. Wang, "Transient oscillations induced by delayed growth response in the chemostat," Journal of Mathematical Biology, vol. 50, no. 5, pp. 489-530, 2005.

[24] S. Ruan and J. Wei, "On the zeros of transcendental functions with applications to stability of delay differential equations with two delays," Dynamics of Continuous, Discrete \& Impulsive Systems. Series A. Mathematical Analysis, vol. 10, no. 6, pp. 863874, 2003.

[25] J. S. Wroblewski and J. G. Richman, “The non-linear response of plankton to wind mixing events-implications for survival of larval northern anchovy," Journal of Plankton Research, vol. 9, no. 1, pp. 103-123, 1987.

[26] J. K. Hale and S. M. Verduyn Lunel, Introduction to FunctionalDifferential Equations, vol. 99, Springer, New York, NY, USA, 1993.

[27] H. Xia, G. S. K. Wolkowicz, and L. Wang, "Transient oscillations induced by delayed growth response in the chemostat," Journal of Mathematical Biology, vol. 50, no. 5, pp. 489-530, 2005.

[28] X. Meng, Q. Gao, and Z. Li, “The effects of delayed growth response on the dynamic behaviors of the Monod type chemostat model with impulsive input nutrient concentration," Nonlinear Analysis: Real World Applications, vol. 11, no. 5, pp. 44764486, 2010. 


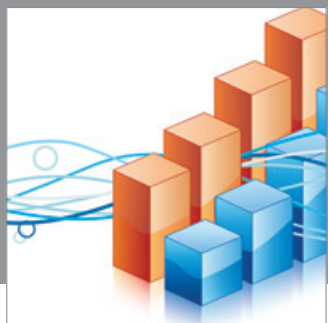

Advances in

Operations Research

mansans

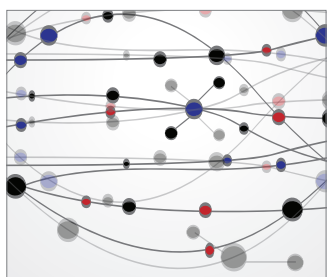

The Scientific World Journal
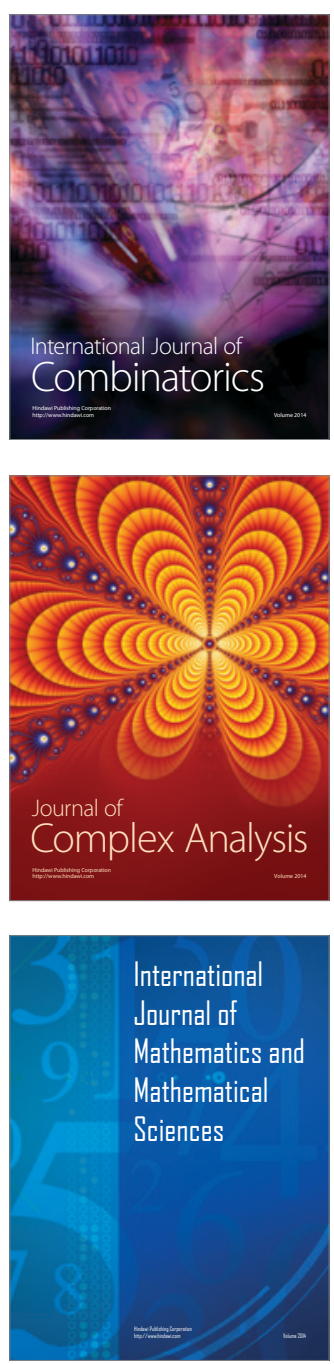
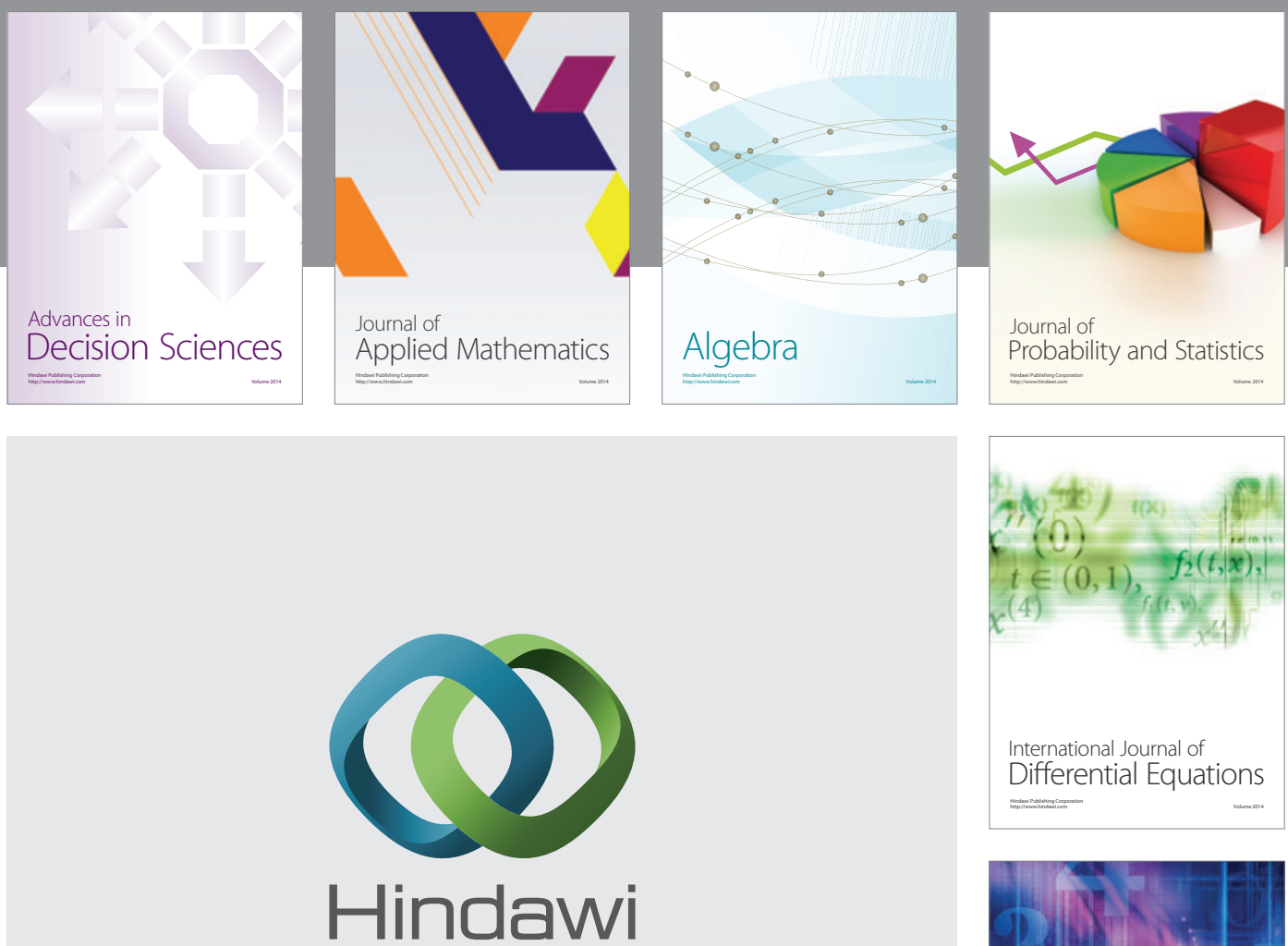

Submit your manuscripts at http://www.hindawi.com
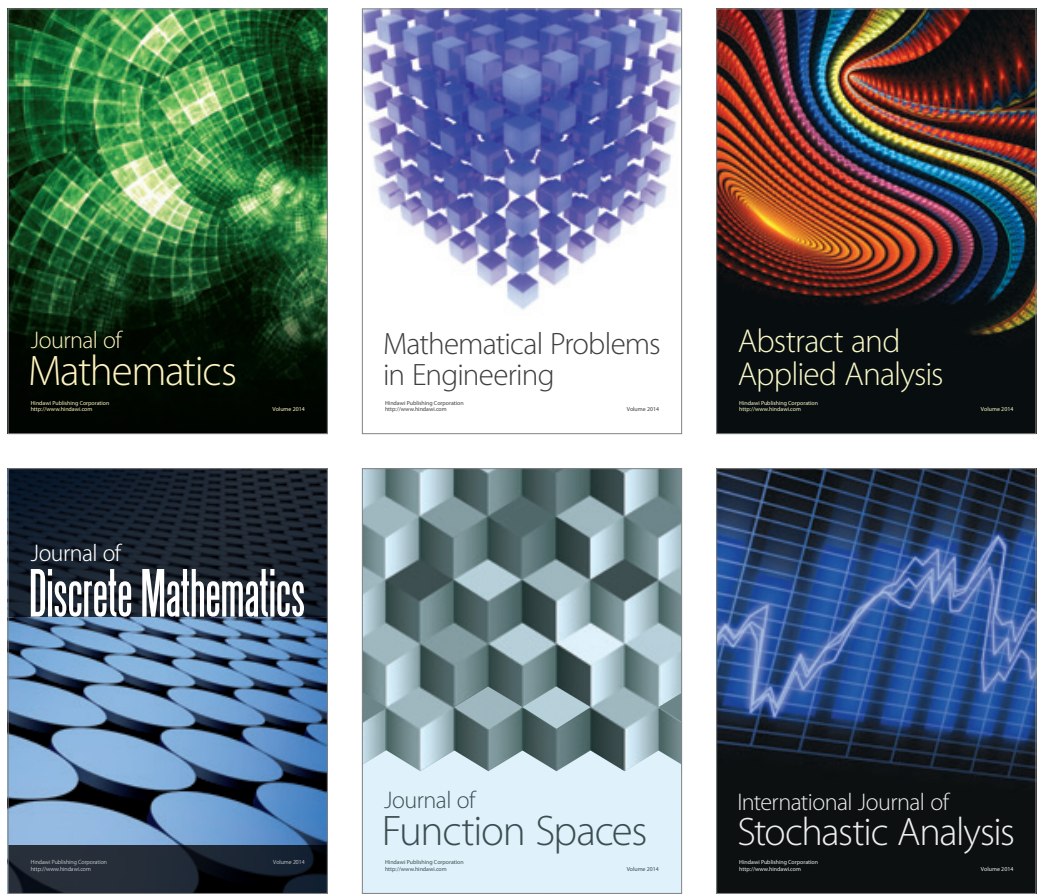

Journal of

Function Spaces

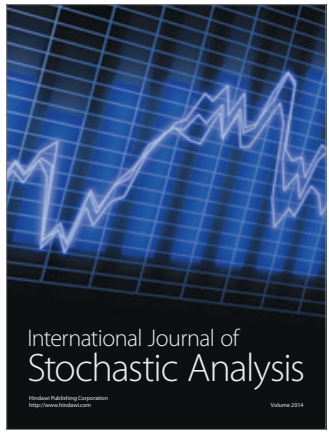

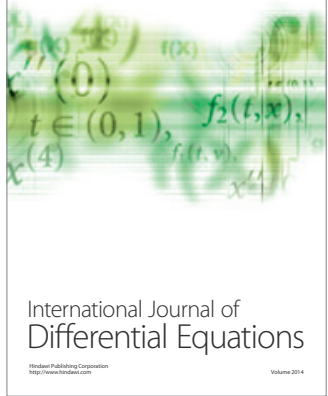
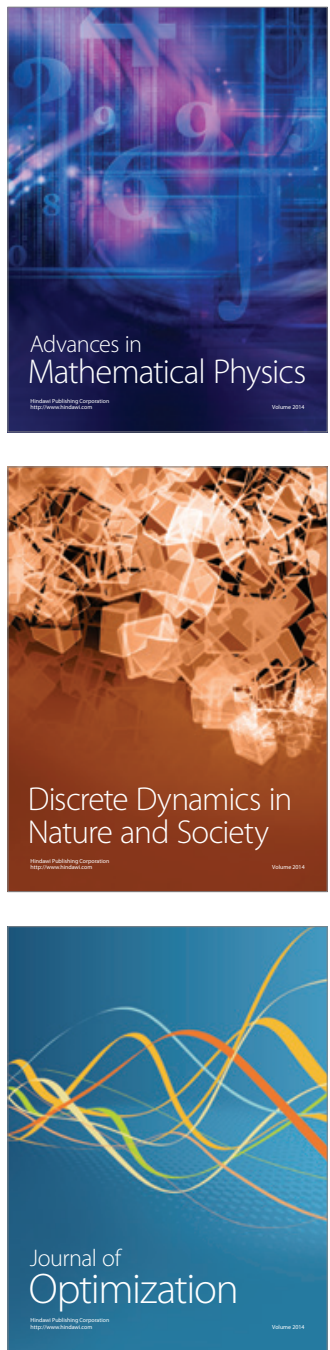